\title{
Excessive closure of the right eye: a new sign of infarction in the territory of the ipsilateral right middle cerebral artery
}

\author{
Shingo Ohkawa, Atsushi Yamadori, Kiyoshi Maeda, Masayasu Tabuchi, Yukio Ohsumi, \\ Etsuro Mori, Takashi Yoshida, Yukihiro Yoneda, Toshiyuki Uehara
}

\begin{abstract}
Neurology Service,
Hyogo Brain and

Heart Center at

Himeji, Japan

S Ohkawa

A Yamadori

K Maeda

M Tabuchi

Abstract

In right middle cerebral territory infarction a new sign, excessive closure of the right eye ipsilateral to the lesion and mild closure of the left eye on command, was noted. The excessive ipsilateral eye closure was not observed on spontaneous eye closure.
\end{abstract}

Y Ohsumi

E Mori

T Yoshida

Y Yoneda

T Uehara

Correspondence to: Shingo Ohkawa, MD

Neurology Service, Hyogo

Brain and Heart Center at

Himeji, 520, Saisho-Ko,

Himeji, 670 Japan.

Received 22 June 1992

and in revised form

September 1992

Accepted 8 October 1992

$(\Im$ Neurol Neurosurg Psychiatry 1993;56:894-896)

A variety of eye abnormalities secondary to hemispheric lesions have been described. These include ptosis, difficulty of initiating eye closure or eye opening, and difficulty of maintaining eye closure or eye opening. ${ }^{1-5} \mathrm{We}$ describe here a new eyelid sign in patients with acute hemispheric infarction in the

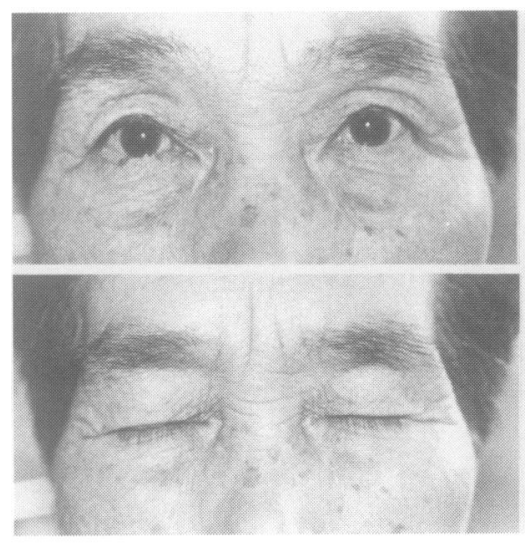

a

Figure 1 Photographs of the eyes of control (a) and left brain damaged patient (b). Top: spontaneous eye opening. Bottom: volitional eye closure. Symmetrical and mild closure of the eyes.
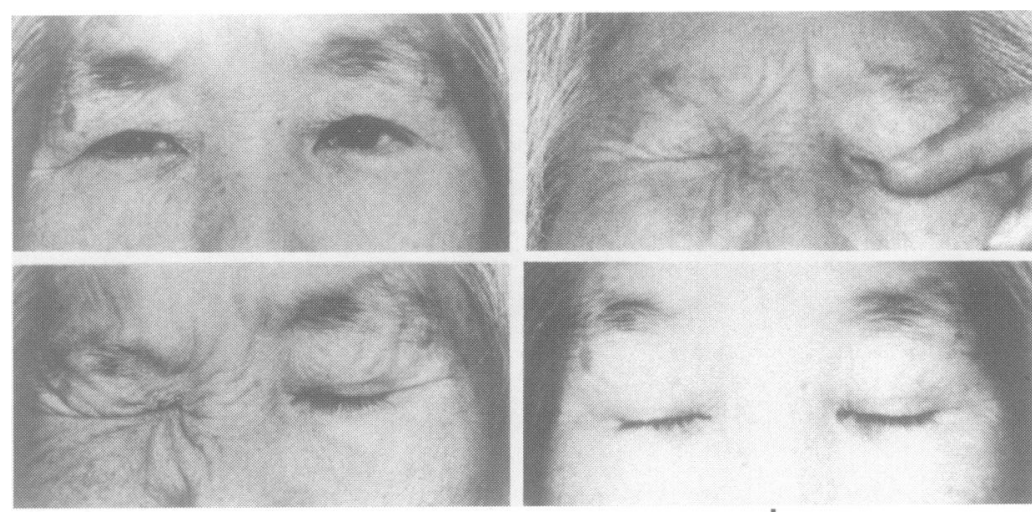

C
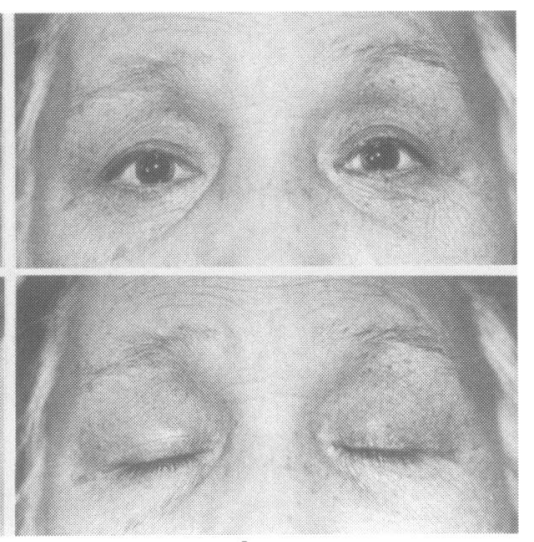

$\mathrm{b}$

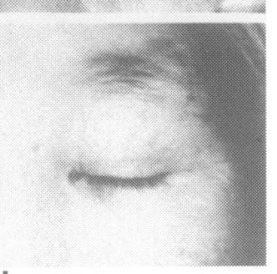

Figure 2 Photographs of the eyes of right brain damaged patient with ipsilateral excessive eye closure. Spontaneous eye opening (c, top) and volitional eye closure (c, bottom). Excessive closure of the right eye and mild closure of the left eye. Volitional eye closure with the left eye closed by the examiner $(d$, top). Closure of the right eye is excessive. Spontaneous eye closure $(d$, bottom). Closure of the eyes is gentle and symmetrical. territory of the right middle cerebral artery (MCA). This sign consists of excessive closure of the right eye ipsilateral to the side of a fresh infarction and mild closure of the contralateral left eye. To our knowledge, this ipsilateral excessive eye closure (IEEC) has not been mentioned in any published reports.

\section{Subjects and methods}

The subjects were 22 patients with acute hemispheric infarction in the territory of the MCA and 10 patients without signs, symptoms, or history of disease involving the brain who were admitted to the neurology service of Hyogo Brain and Heart Centre at Himeji from July 1987 to February 1992. Fourteen were right brain damaged (RBD) and eight were left brain damaged (LBD). Ten nonbrain damaged patients served as controls. All were right handed. Patients with severe aphasia or confusion who did not respond to commands were not included in the brain damaged groups.

The RBD group included eight men and six women, the LBD group six men and two women, and the control group five men and five women. The mean age was 62.5 years (SD 10.9) for the RBD group, 59.6 (SD 13.1) for the LBD group, and 66.9 (SD 10.5) for the control group.

When the eyes were open subjects were requested to close them on verbal command. When necessary, the examiner accompanied verbal instructions with a demonstration of eye closing movement. The degree and asymmetry of the eye closing behaviour were carefully analysed, according to photographs of the eyes.

\section{Results}

All the 22 brain damaged patients had hemiplegia including the lower face on the contralateral side and additional cortical signs. All were somewhat obtunded, but cooperative and able to comply with commands to close the eyes without any difficulty.

When asked to close the eyes, all of the 10 control patients closed them lightly. No excessive contraction of the muscles around the eyes were observed. The upper portion of the facial muscles, the eyebrows and the upper eyelids appeared almost symmetrical (fig 1a).

Seven of the RBD group and all of the 

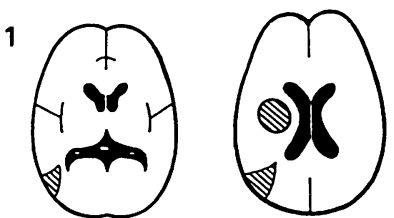

2
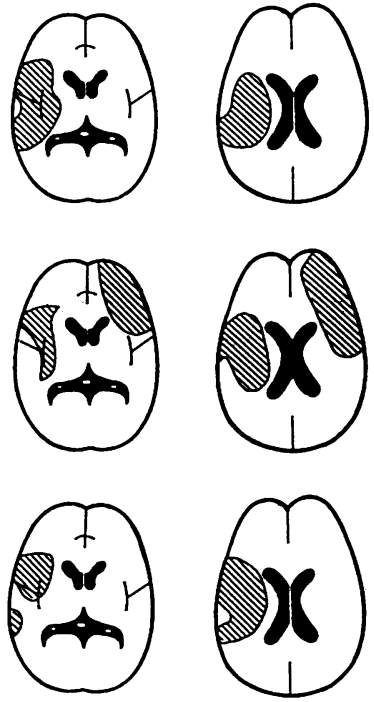

A
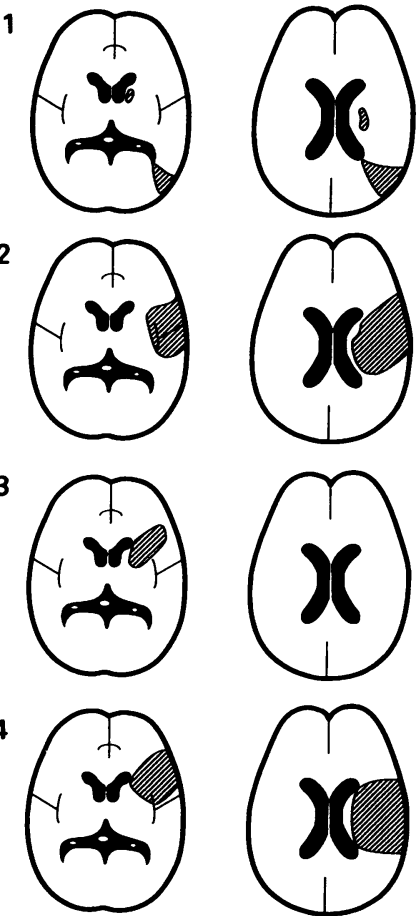

$\mathbf{R}$
5
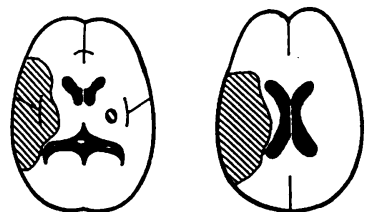

6

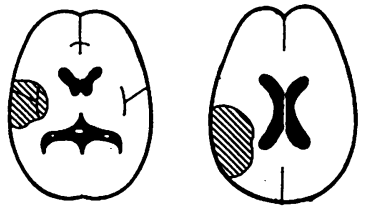

7
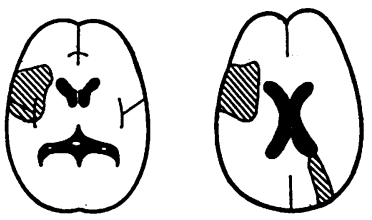

L

$\mathbf{R}$

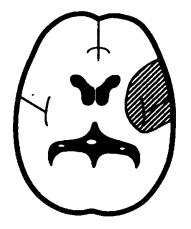

6
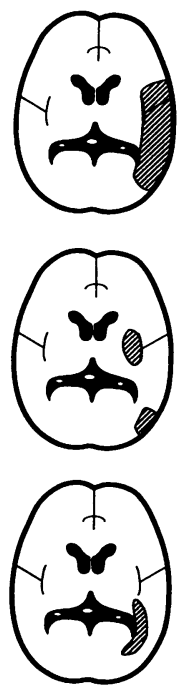
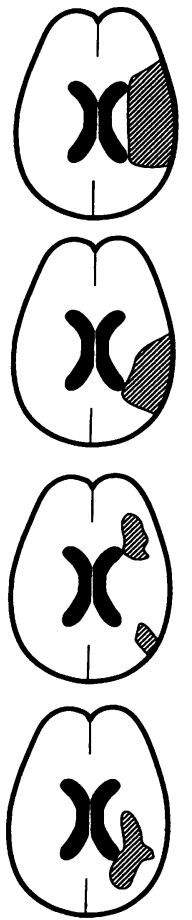

L $\mathbf{R}$
LBD group closed the eyes symmetrically and lightly exactly like the control group (fig 1b). However, seven of the RBD patients closed the ipsilateral right eye much more tightly than the left one (fig 2c), producing remarkable asymmetry of the upper face. This was characterised by the squeezed eyelid with the furrowed skin, the lowered eyebrow, and the hidden cilia.

This ipsilateral excessive eye closure (IEEC) had additional common features. All IEEC patients could not control the degree of eye closure on the right side voluntarily even when they were specifically asked to close it gently. When the left eye was covered or closed by the examiner (fig $2 \mathrm{~d}$, top), the right eye still showed excessive closure. Involuntary eye closing behaviour was normal and symmetrical, demonstrating automaticovoluntary dissociation. In sleep their eyes were closed gently and symmetrically (fig $2 \mathrm{~d}$, bottom). On spontaneous blinking the eyes were opened and closed symmetrically. There was no visible blepharospasm. Reflex blepharospasm was not elicited when the examiner attempted to hold their eyelids apart. ${ }^{6}$ There was also no other involuntary facial movement such as focal seizure or myokymia.

In all seven cases the excessive closure of the right eye was observed within a few days after the onset of a stroke. In four patients (cases 4-7) this abnormality subsided within a few weeks but in two patients it persisted for a few years (cases 1 and 2). One patient died soon after hospitalisation.

The surface EMG of the orbicularis oculi muscles was performed in two patients (cases 1 and 2). When closing the eyes on command, a remarkable burst of orbicularis activity was observed on the right side in both cases, but not on the left side.

$\mathrm{CT}$ scans of the brain were available for all the brain damaged patients. All the RBD with IEEC patients had new lesions in the perisylvian area in the MCA territory (fig 3a). Three had additional left cerebral infarctions which were old. The distribution of lesions in the LBD appeared grossly similar to that in the RBD with IEEC (fig 3b). It remained to be seen whether there was any difference in the RBD with and without IEEC.

Clinical features of the RBD with IEEC are summarised in the table.
B

Figure 3 Schematic representation of $C T$ lesions. $A: R B D$ patients with IEEC. Number represents patient number as summarized in table. $B$ : $L B D$ patients.
Table Clinical features of patients with IEEC

\begin{tabular}{|c|c|c|c|c|c|c|c|c|c|c|c|c|}
\hline Case & Sex & Age & $H P$ & $H S$ & $H A$ & $I G R$ & USN & Ext & Ano & $M I$ & Aph & $L H L$ \\
\hline 1 & $\mathbf{F}$ & 73 & + & + & + & + & + & + & + & + & - & $\mathbf{N}$ \\
\hline 2 & $\mathbf{M}$ & 81 & + & + & + & & + & + & + & + & - & $\mathbf{N}$ \\
\hline 3 & $\mathbf{M}$ & 68 & + & & & & + & & & & + & frontal infarction \\
\hline 4 & $\mathbf{F}$ & 55 & + & + & + & & + & + & + & + & - & \\
\hline 5 & $\mathbf{M}$ & 51 & + & + & + & & + & + & + & + & - & $\begin{array}{l}\text { small infarction } \\
\text { (basal) }\end{array}$ \\
\hline 6 & $\mathbf{M}$ & 66 & + & + & + & - & + & + & + & + & - & \\
\hline 7 & $\mathbf{M}$ & 72 & + & - & + & & + & + & + & + & - & $\begin{array}{l}\text { water shed } \\
\text { infarction }\end{array}$ \\
\hline
\end{tabular}

IEEC: ipsilateral excessive eye closure; HP: left hemiparesis including the lower face; HS: left hemisensory deficit; HA: left hemianopsia; IGR: insilateral grasp reflex; USN: left unilateral spatial neglect; Ext: extinction on double-simultaneous-stimula Ano: anosognosia for the left hemiplegia; MI: motor impersistence; Aph: aphasia; LHL: left hemispheric lesion by the CT scans; $\mathbf{N}$ : none 


\section{Discussion}

Asymmetrical and excessive eye closure of the right eye, observed in new stroke patients with right MCA infarctions, has not been described to our knowledge. This ipsilateral excessive eye closure (IEEC) was present in seven of the $14 \mathrm{RBD}$ patients personally cared for by one of the authors (SO), but not in any LBD patients and controls. Because it was only elicitable when patients were asked to close their eyes we would like to call this sign "volitional IEEC."

It is clear that overcontraction of the orbicularis oculi muscles on the right side was a cause of this sign. It is true that normal persons can intentionally produce asymmetrical excessive eye closure, or it can occur as a habit. IEEC, however, cannot be interpreted as a sign of such physiological movements, because it was uncontrollable when the patients were conscious. Also, this phenomenon cannot be an autonomous eyelid movement triggered by mild closure of the left eye, because it appeared even when the left eye was closed passively. It seems that the sign was pathological and intimately related with the right hemisphere damage.

Volitional eye closure is controlled by the pyramidal system. The fibres in this system descend from the precentral motor cortex to the facial nuclei. ${ }^{7}$ The distribution of the fibres is bilateral but greater to the contralateral nucleus. ${ }^{8910}$

We have recently argued that the right hemisphere contains a higher motor control mechanism for smooth and symmetrical eye closure which exerts its influence on intentional eye closure by modulating the activity of the motor cortex subserving eye closure. ${ }^{11}$ If this control mechanism and the motor cortex were damaged by an appropriately placed lesion in the right hemisphere, the physiologically dominant contralateral cortical innervation to the facial nuclei would become enhanced, resulting in overcontraction of the contralateral right orbicularis oculi muscles. If, however, the left hemisphere motor cortex was damaged, this right hemispheric control mechanism could produce normal bilateral and symmetrical eye closure through the right motor cortex.

Why does this phenomenon occur in some RBD patients and not in others, and why does it persist in some individuals? We speculate that sparing of this higher mechanism in case of the right hemispheric motor cortex damage would permit its normal activity via the corpus callosum through the left hemisphere motor cortex. Persistence of the sign may mean the permanent damage to this hypothetical apparatus and the right motor cortex.

1 Caplan LA. Ptosis. $f$ Neurol Neurosurg Psychiatry 1974 37:1-7.

2 Lewandowsky M. Ueber Apraxie des Lidschluss. Berlin Klin Wochenschr 1907;44:921-3.

3 Goldstein JE, Cogan DG. Apraxia of lid opening. Arch Ophthalmol 1965;73:155-9.

4 Berlin L. Compulsive eye opening and associated phenomenon. Arch Neurol 1955;73:597-601.

5 Fisher CM. Left hemiplegia and motor impersistence. $f$ Nerv Ment Dis 1956;123:201-18.

6 Fisher CM. Reflex blepharospasm. Neurology 1963;13: 77-8.

7 Penfield W, Rasmussen T. The cerebral cortex of man. New York: Hafner, 1968:51-76.

8 Leyton ASF, Sherrington CS. Observations of the excitable cortex of the chimpanzee, orang-utang, and gorilla. $Q \mathcal{F}$ Exp Physiol 1917;11:135-222.

9 Crosby EC, DeJonge BR. Experimental and clinical studies of the central connections and central relations of the facial nerve. Ann Otol 1963;72:735-55.

10 Walsh FB, Hoyt WF. The ocular motor system: anatomy, physiology and topographic diagnosis. In: Walsh FB, physiology and topographic diagnosis. In: Walsh FB, eds. Clinical neuro-ophthalmology 3rd

11 Ohkawa S, Yamadori A. Right-left dissociation of voluntary eyelid closure following right middle cerebral artery infarction. Clin Neurol (Tokyo) 1990;30:294-8. 